\title{
NACO Ks, Lp and M band imaging of NGC 1068
}

\author{
Damien Gratadour ${ }^{1,3}$, Daniel Rouan ${ }^{1}$, Eric Gendron ${ }^{1}$, \\ Francois Lacombe ${ }^{1}$ and Yann Clénet ${ }^{2}$ \\ ${ }^{1}$ Observatoire de Paris-Meudon / LESIA, 92195 Meudon, France \\ email:damien.gratadour@obspm.Fr \\ ${ }^{2}$ ESO, Karl-Schwarzschild-Straße 2, Garching, Germany \\ ${ }^{3}$ ONERA / DOTA, BP 72, 92322 Châtillon cedex, France
}

\begin{abstract}
We present near-IR, diffraction limited imaging of the nucleus of NGC 1068 obtained with NAOS-CONICA at VLT. These observations provide an accute view on the distribution of hot dust in the vicinity of the central source, at a 0.1 " resolution. The use of a new type of coronagraph allowed us to detect and identify new structures at $\mathrm{K}$, to calculate more accurate color indices, and derive the state of the dust in this very complex morphology. We also used deconvolution on images aquired in other bands to get an accute view of these structures.
\end{abstract}

\section{Coronagraphic observations}

We used a 4 quadrant phase mask recently installed on NAOS-CONICA at VLT. The images in Fig. 1 represent a total integration time of 100 sec. We also aquired images of a reference star, that we substracted from the object to obtain the images presented in the right panel of Fig. 1, making the northern knots and central structures more conspicuous. These images put evidence on the same structures discovered in Lp and M (Rouan et al. 2004) allowing us to derive color indices. We confirm the color temperature of 550 $\mathrm{K}$ for these knots $(\mathrm{Ks}-\mathrm{L}=4.7$ and $\mathrm{L}-\mathrm{M}=1.7)$, meaning that if they are heated by the central source, they should be transiently heated and so be Very Small Grains. In such a shocked and UV illuminated region, the best VSG candidates are nanodiamonds. We also detect new structures located a hundred pc S-W of the central source, where the emission seems to be distributed in a series of three (maybe four) very regular arclets. Such morphology suggests a very peculiar mechanism, one interpretation could be the effect of an AGN pulsating wind (Elvis et al. 2000). Closer to the core, we find very peculiar sub-structures that have not been interpreted yet.

\section{Deconvolution : process, results and discussion}

We use the MISTRAL algorithm (ONERA, Conan et al. 1998), to deconvolve images obtained in other bands (Rouan et al. 2004). It uses a regularized Maximum A Posteriori approach with reparametrization by a positivity constraint. We use a new type of regularization, based on the estimation of the object Power Spectral Density by a Maximum Likelihood algorithm. Results are presented in Fig. 2. These images show a very complex but structured hot dust distribution around the core of NGC 1068, and the use of coronagraphy allowed us to detect some new structures closer to the nucleus. We interpret the observed structures as well in Ks, Lp and M, about 40 pc north of the nucleus, as hydrodynamic instabilities in the ISM induced by the jet. The deconvolved data show more precisely their size and morphology, and the photometry is in agreement with previous measurements (i.e. on non-deconvolved data). 


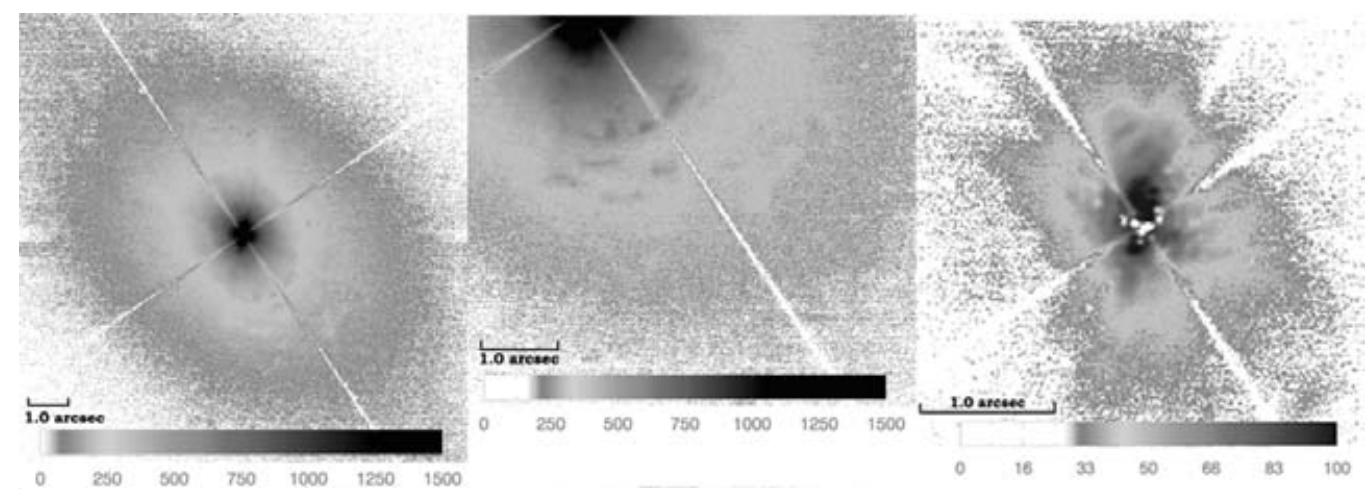

Figure 1. Coronagraphic image of NGC 1068 with a log-scale representation and different color scales to show every structure. North is up and east is left. Left : the whole field : 11.7"x 11.7". Middle: a zoom south west to show the arclets. Right : PSF substracted coronagraphic images showing the structures close to the core.
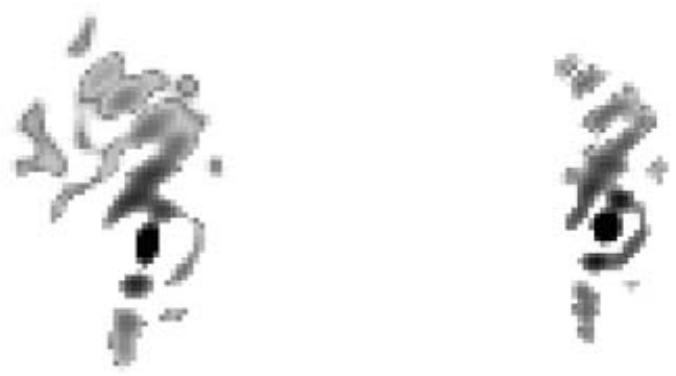

a

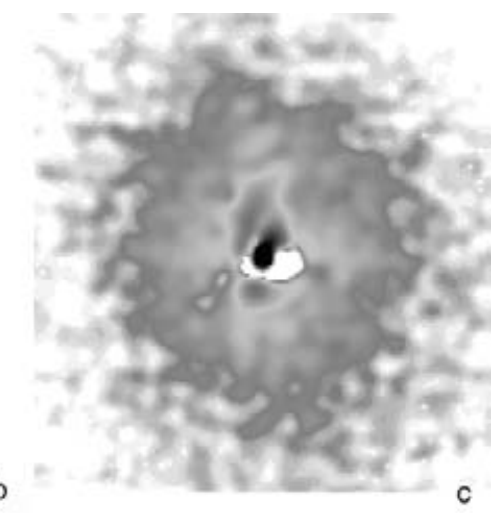

Figure 2. Deconvolved images using MISTRAL. From left to rigth : M band (a), L band (b), Ks band (c). We used a log-scale representation.

\section{Conclusion}

By use of either new instrumental methods and new numerical methods, we confirmed the presence and nature of new structures in NGC 1068. We also found new structures south of the nucleus that would testify for specific mechanisms (nuclear winds for instance) in the core of this active galaxy.

\section{References}

Rouan, D., et al. 2004, A\&A, 417, L1

Conan, J.-M., et al. 1998, Appl Opt, 37, 4614

Elvis, M., et al. 2000, A\&A, 545, 63 